\title{
Genetic variants in DKK1 linked to severity of joint damage in RA
}

"Only part of the variance in joint destruction in patients with RA can be explained by currently known factors", explains Diederik de Rooy from Leiden University, The Netherlands. "Identifying new genetic risk factors may yield new insights into the pathophysiology of the disease." This motivation led to the publication in Annals of the Rheumatic Diseases of research showing that patients with RA carrying risk alleles of genetic variants in $D K K 1$ have increased serum protein levels of its product, Dickkopf-1 (DKK1), and more severe joint destruction than patients without these genetic factors.

DKK1 is a negative regulator of the Wnt- $\beta$-catenin signalling pathway; components of this pathway, including those encoded by DKK1, SOST, LRP5 and KREMEN1, have known roles in osteoblast differentiation and in joint remodelling. On the basis of previous research, the authors hypothesized that

\section{The DKK1 SNP with the strongest association with joint damage ... resulted in higher serum levels of DKK1... 77}

single nucleotide polymorphisms (SNPs) in these genes might be associated with joint damage in RA. Their study involved two phases, as de Rooy explains: "In the first phase, SNPs tagging these genes were typed in patients included in the Leiden Early Arthritis Clinic and studied for a relation to the progression of joint destruction. In the second phase, the SNPs that showed a significant association in phase 1 were typed in three replication cohorts. Subsequently, a meta-analysis on the four cohorts was performed."

In the Leiden cohort, 6 DKK1, 10 LRP5, 3 SOST and 1 KREMEN1 SNPs were associated with rate of joint destruction, as assessed radiographically over a period of 7 years. The meta-analysis performed in phase 2 showed all 6 DKK1 SNPs to be significantly associated with progression of joint damage; after correcting for multiple testing, 3 remained significant (rs1896368, rs1896367 and rs1528873). No SNPs in the other genes retained significance after correction for multiple testing.

The DKK1 SNP with the strongest association with joint damage progression (rs1896368) resulted in higher levels of serum DKK1 protein than seen in patients of other genotypes. de Rooy concludes, "This research provides a new line of evidence for the Wnt- $\beta$-catenin signalling pathway, particularly DKK1, as a mediator of RA joint damage."

Jenny Buckland

Original article de Rooy, D. P. C. et al. Genetic studies on components of the Wnt signalling pathway and the severity of joint destruction in rheumatoid arthritis. Ann. Rheum. Dis. doi:10.1136/annrheumdis-2012-202184 